\title{
Emergent Universe with Exotic Matter in Brane World Scenario
}

\author{
Ujjal Debnath $^{1 *}$ and Subenoy Chakraborty ${ }^{2 \dagger}$ \\ ${ }^{1}$ Department of Mathematics, \\ Bengal Engineering and Science University, \\ Shibpur, Howrah-711 103, India \\ ${ }^{2}$ Department of Mathematics, \\ Jadavpur University, Kolkata 700 032, India.
}

(Dated: November 7, 2018)

\begin{abstract}
In this work, we have examined the emergent scenario in brane world model for phantom and tachyonic matter. For tachyonic matter field we have obtained emergent scenario is possible for closed, open and flat model of the universe with some restriction of potential. For normal scalar field the emergent scenario is possible only for closed model and the result is identical with the work of Ellis et al [2], but for phantom field the emergent scenario is possible for closed, open and flat model of the universe with some restriction of potential.
\end{abstract}

PACS numbers:

In recent years to avoid the big-bang singularity, attempts have been made in the perspective of quantum gravity (especially in loop formalism) as well as in classical general relativity. The idea of emergent universe is the result of the search for singularity free inflationary model in general relativity. An emergent universe is a model universe without a singularity, which is ever exiting and has an almost static behaviour in the infinite past $(t \rightarrow-\infty)$ and evolves into an inflationary stage. Also an extension of the original Lemaitre-Eddington model is termed as emergent universe.

Since 1967, there was solution in GR having no big-bang singularity. Harrison [1] described a model of a closed universe with radiation, which as infinite past coincides with Einstein static model. Then after a long gap (of about 40 years) similar kind of model was discovered by Ellis et al [2,3]. They obtained closed model of the universe with a minimally coupled scalar field having self-interacting potential together with a non-interacting perfect fluid having equation of state $p=w \rho\left(-\frac{1}{3} \leq w \leq 1\right)$. Instead of finding exact analytical solutions, they studied the asymptotic behaviour to characterize the emergent model. Subsequently, Mukherjee et al [4] obtained solutions of semiclassical Einstein equations (for the Starobinsky model [4]) for flat FRW space-time and examined the features of emergent universe. Then a general framework for emergent universe model showing non-singular (i.e., geodesically complete) inflationary solution was proposed by Mukherjee et al [5] where a part of the matter is in exotic form. Also Campo et al [6] have studied an emergent universe model for self-interacting Brans-Dicke theory. Recently, Banerjee et al [7] have shown a model of emergent universe in brane world scenario, while Debnath [8] has presented an emergent universe model for exotic matter in the form of phantom or tachyonic field.

In this work, we extend the idea of Debnath [8] to brane scenario. According to Randall and Sundram [9] our universe is assumed to be a 3-brane embedded in a 5D bulk space-time. All standard model fields are confined on the brane, only gravity can propagate in the whole space-time. The Einstein equations on the brane get modified due to the existence of extra dimension. In RS II [10] model the effective equations of motion on the 3 -brane embedded in $5 \mathrm{D}$ bulk having $Z_{2}$-symmetry are given by

$$
{ }^{(4)} G_{\mu \nu}=-\Lambda_{4} q_{\mu \nu}+\kappa_{4}^{2} \tau_{\mu \nu}+\kappa_{5}^{4} \Pi_{\mu \nu}-E_{\mu \nu}
$$

where

$$
\kappa_{4}^{2}=\frac{1}{6} \lambda \kappa_{5}^{4}
$$

\footnotetext{
* ujjaldebnath@yahoo.com, ujjal@iucaa.ernet.in

† schakraborty@math.jdvu.ac.in,subenoyc@yahoo.co.in
} 


$$
\Lambda_{4}=\frac{1}{2} \kappa_{5}^{2}\left(\Lambda_{5}+\frac{1}{6} \kappa_{5}^{2} \lambda^{2}\right)
$$

and

$$
\Pi_{\mu \nu}=-\frac{1}{4} \tau_{\mu \alpha} \tau_{\nu}^{\alpha}+\frac{1}{12} \tau \tau_{\mu \nu}+\frac{1}{8} q_{\mu \nu} \tau_{\alpha \beta} \tau^{\alpha \beta}-\frac{1}{24} q_{\mu \nu} \tau^{2}
$$

and $E_{\mu \nu}$ is the electric part of the $5 \mathrm{D}$ Weyl tensor. Here $\kappa_{5}, \Lambda_{5}, \tau_{\mu \nu}$ and $\Lambda_{4}$ are respectively the $5 \mathrm{D}$ gravitational coupling constant, 5D cosmological constant, the brane tension (vacuum energy), brane energymomentum tensor and effective 4D cosmological constant. Choosing our 3-brane as FRW universe i.e.,

$$
d s^{2} \equiv q_{\mu \nu} d x^{\mu} d x^{\nu}=d t^{2}-a^{2}(t)\left[\frac{d r^{2}}{1-k r^{2}}+r^{2}\left(d \theta^{2}+\sin ^{2} \theta d \phi^{2}\right)\right]
$$

the explicit form of the above modified Einstein equations are

$$
3 H^{2}+\frac{3 k}{a^{2}}=\Lambda_{4}+\kappa_{4}^{2} \rho+\frac{\kappa_{4}^{2}}{2 \lambda} \rho^{2}+\frac{6}{\lambda \kappa_{4}^{2}} \mathcal{U}
$$

and

$$
2 \dot{H}+3 H^{2}+\frac{k}{a^{2}}=\Lambda_{4}-\kappa_{4}^{2} p-\frac{\kappa_{4}^{2}}{2 \lambda} \rho p-\frac{\kappa_{4}^{2}}{2 \lambda} \rho^{2}-\frac{2}{\lambda \kappa_{4}^{2}} \mathcal{U}
$$

where matter in the bulk is chosen as the perfect fluid having energy-momentum tensor

$$
\tau_{\mu \nu}=(\rho+p) u_{\mu} u_{\nu}+p q_{\mu \nu}
$$

The matter density $\rho$ on the brane satisfies the continuity equation

$$
\dot{\rho}+3 H(\rho+p)=0
$$

and the dark radiation $\mathcal{U}$ obeys

$$
\dot{\mathcal{U}}+4 H \mathcal{U}=0
$$

In the present work, we consider exotic matter on the brane in the form of phantom field or tachyonic field and examine the possibility of an emergent universe. For emergent model of the universe, the scale factor may be chosen as $[5,8]$

$$
a=a_{0}\left(\beta+e^{\alpha t}\right)^{n}
$$

where $a_{0}, \alpha, \beta$ and $n$ are positive constants. So the Hubble parameter and its derivatives are given by

$$
H=\frac{n \alpha e^{\alpha t}}{\left(\beta+e^{\alpha t}\right)}, \dot{H}=\frac{n \beta \alpha^{2} e^{\alpha t}}{\left(\beta+e^{\alpha t}\right)^{2}}, \ddot{H}=\frac{n \beta \alpha^{3} e^{\alpha t}\left(\beta-e^{\alpha t}\right)}{\left(\beta+e^{\alpha t}\right)^{3}}
$$

Here $H$ and $\dot{H}$ are both positive, but $\ddot{H}$ changes $\operatorname{sign}$ at $t=\frac{1}{\alpha} \log \beta$. Thus $H, \dot{H}$ and $\ddot{H}$ all tend to zero as $t \rightarrow-\infty$. On the other hand as $t \rightarrow \infty$ the solution gives asymptotically a de Sitter universe.

For the above choice of scale factor, the deceleration parameter $q$ can be simplified to the form 


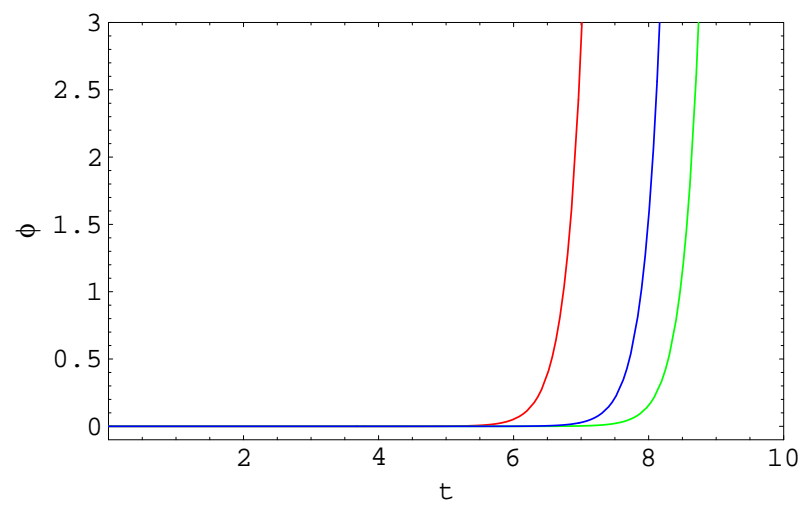

Fig.1

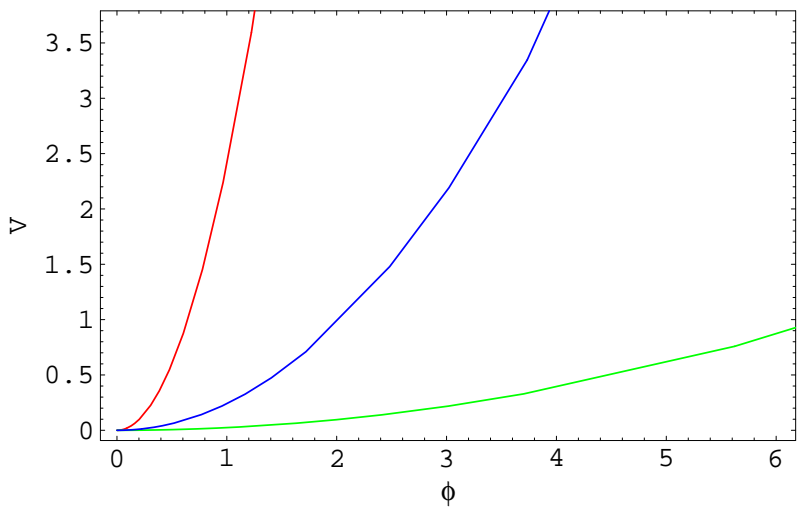

Fig.2

Fig. 1 represents the variation of $\phi$ against $t$ for normalizing the constants $\alpha=2, \beta=4$ and Fig. 2 shows the variation of $V$ against $\phi$ for normalizing the constants $\alpha=2, \beta=4, n=4, a_{0}=1, \kappa=1, \lambda=10, \Lambda=0$ in phantom field for closed (red line), flat (blue line) and open (green line) universe.

$$
q=-1-\frac{\beta}{n e^{\alpha t}}
$$

- Normal or Phantom field: The phantom field has negative kinetic term so that the ratio between pressure and energy density is always less than -1 . The explicit form of energy density and pressure are [11]

$$
\rho=\rho_{\phi}=\frac{\delta}{2} \dot{\phi}^{2}+V(\phi)
$$

and

$$
p=p_{\phi}=\frac{\delta}{2} \dot{\phi}^{2}-V(\phi)
$$

where $\delta=+1$ corresponds to usual scalar field and $\delta=-1$ represents phantom scalar field. Now subtracting field equations (6) and (7) and using the expressions for $\rho$ and $p$ from (14) and (15), we have

$$
\kappa_{4}^{2} \delta \dot{\phi}^{2}=-2 \dot{H}+\frac{2 k}{a^{2}}-\frac{\kappa_{4}^{2}}{\lambda}\left(\frac{\dot{\phi}^{4}}{4}-V^{2}(\phi)\right)-\frac{\kappa_{4}^{2}}{\lambda} \rho^{2}-\frac{8}{\lambda \kappa_{4}^{2}} \mathcal{U}
$$

This is a quadratic in $\dot{\phi}^{2}$ and we get

$$
\dot{\phi}^{2}=\frac{-B+\sqrt{B^{2}-4 A C}}{2 A}
$$

with $A=\frac{\kappa_{4}^{2}}{2 \lambda}>0, B=\kappa_{4}^{2} \delta\left(1+\frac{V(\phi)}{\lambda}\right), C=2 \dot{H}-\frac{2 k}{a^{2}}+\frac{8}{\lambda \kappa_{4}^{2}} \mathcal{U}$.

We shall now state the various possibilities in the form of propositions:

Proposition I: For normal scalar field the emergent scenario is possible only for the closed model of the universe and we must have

$$
2 \dot{H}+\frac{8}{\lambda \kappa_{4}^{2}} \mathcal{U}<\frac{2}{a^{2}}
$$




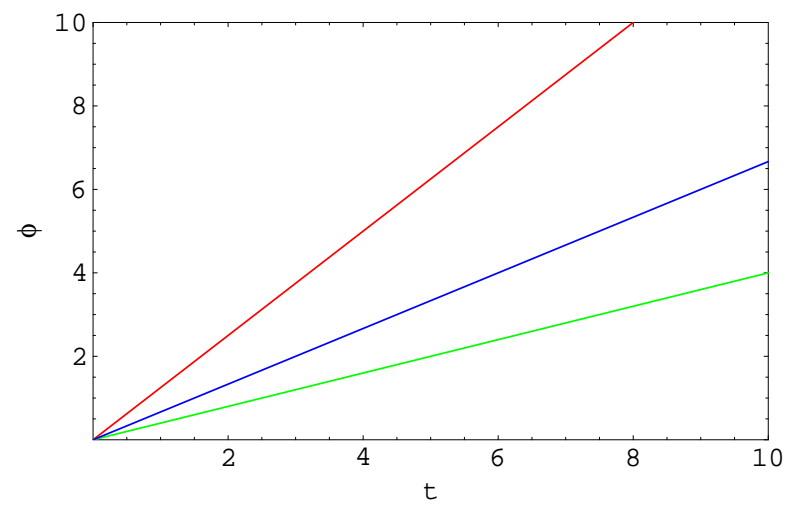

Fig.3

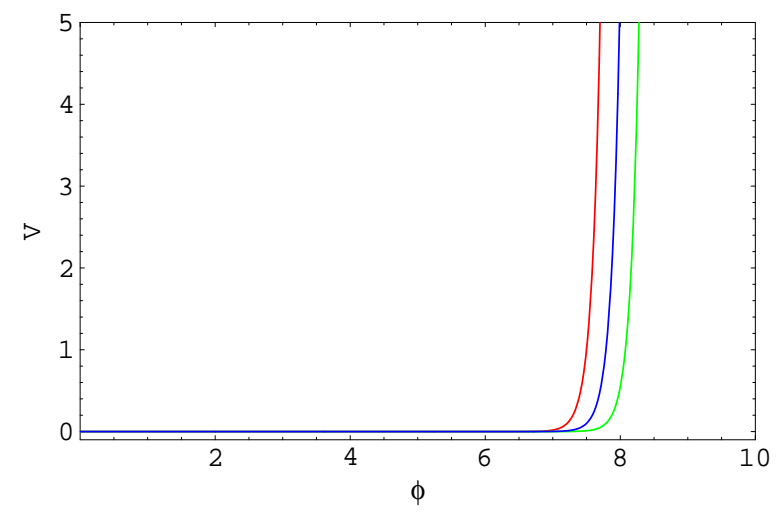

Fig.4

Fig. 3 represents the variation of $\phi$ against $t$ for normalizing the constants $\alpha=2, \beta=4$ and Fig. 4 shows the variation of $V$ against $\phi$ for normalizing the constants $\alpha=2, \beta=4, n=4, a_{0}=1, \kappa=1, \lambda=10, \Lambda=0$ in phantom tachyonic field for closed (red line), flat (blue line) and open (green line) universe.

This clearly follows from the expression of $\dot{\phi}^{2}$ in equation (17). Note that for normal scalar field $B>0$ and hence to make the r.h.s. of (17) to be positive definite we must have $C<0$. This is possible only when $k>0$ (i.e., closed model) and we have the given restriction. Note also that here the dark radiation term is against the formation of an emergent universe.

Proposition II: For phantom field, the emergent scenario is possible for any model (closed, open or flat) of the universe, however for open or flat model the potential is restricted by the relation

$$
V(\phi)>\frac{2 \sqrt{\lambda}}{\kappa_{4}}\left(\dot{H}-\frac{k}{a^{2}}+\frac{4}{\lambda \kappa_{4}^{2}} \mathcal{U}\right)^{\frac{1}{2}}-1
$$

In case of phantom scalar field $(\delta=-1) B<0$, so the r.h.s. of (17) will be positive definite for all values of $C$ provided $B^{2}>4 A C$ for $C>0$ and it results the above restriction for $V(\phi)$.

Fig. 1 and 2 show the variation of $\phi$ against $t$ and the variation of $V$ against $\phi$ respectively for normalizing the constants $\alpha=2, \beta=4, n=4, a_{0}=1, \kappa=1, \lambda=10, \Lambda=0$ for phantom field for closed (red line), flat (blue line) and open (green line) universe. Fig. 1 shows the early time $\phi$ is almost constant and then increases very sharply at finite time, while $V$ continuously increases with $\phi$.

- Tachyonic field: The Lagrangian for the tachyonic field $\psi$ having potential $U(\psi)$ is given by [12]

$$
\mathcal{L}=-U(\psi) \sqrt{1-\epsilon \dot{\psi}^{2}}
$$

where as before $\epsilon=+1$ represents the normal tachyon and $\epsilon=-1$ corresponds to phantom tachyon. The expressions for energy density and pressure are

$$
\rho=\rho_{\psi}=\frac{U(\psi)}{\sqrt{1-\epsilon \dot{\psi}^{2}}}
$$

and

$$
p=p_{\psi}=-U(\psi) \sqrt{1-\epsilon \dot{\psi}^{2}}
$$


Due to the above complicated forms for $\rho$ and $p$. Similar to the previous case, it is not possible to find an expression for $\dot{\psi}^{2}$ from the field equations (or any combination of them). However, from the field equation (7) we may write

$$
1-\epsilon \dot{\psi}^{2}=\frac{1}{\kappa_{4}^{2} \rho\left(1+\frac{\rho}{\lambda}\right)}\left[2 \dot{H}+3 H^{2}+\frac{k}{a^{2}}-\Lambda_{4}+\frac{\kappa_{4}^{2}}{2 \lambda} \rho^{2}+\frac{2}{\lambda \kappa_{4}^{2}} \mathcal{U}\right]
$$

The expression for the Lagrangian or $\rho, p$ shows that the r.h.s. of equation (23) must be positive definite and this is possible for all values of $k$ i.e., for open, closed and flat model of the universe. Thus it is possible to have emergent scenario for any model (closed, open or flat) of the universe with tachyonic field as the matter content, provided

$$
2 \dot{H}+3 H^{2}+\frac{\kappa_{4}^{2}}{2 \lambda} \rho^{2}+\frac{2}{\lambda \kappa_{4}^{2}} \mathcal{U}>\Lambda_{4}-\frac{k}{a^{2}}
$$

Fig. 3 and 4 show the variation of $\phi$ against $t$ and the variation of $V$ against $\phi$ respectively for normalizing the constants $\alpha=2, \beta=4, n=4, a_{0}=1, \kappa=1, \lambda=10, \Lambda=0$ for phantom tachyonic field for closed (red line), flat (blue line) and open (green line) universe. Here $\phi$ increases with constant slope, while $V$ remains almost constant for a wide range of $\phi$ and then increases very sharply.

Thus in the present work, we have shown emergent universe in brane scenario with 5D bulk energy in the form of a cosmological constant and both standard and exotic matter in brane. For normal scalar field, the emergent scenario is possible only for closed model and the result is same as that of Ellis et al [2] and Debnath [8] in GR. In brane world scenario, the emergent model is possible with phantom matter for all values of the curvature parameter $k$ and there is a realization on the potential function in open and flat cases. In GR, Debnath [8] has obtained emergent model with normal tachyonic field only for closed universe but here in brane scenario, it is possible for all $k$. Here the correction term quadratic to the energy-momentum tensor plays a crucial role for emergent scenario. Finally, we mention that the emergent model of Banerjee et al [7] in brane scenario, they have chosen the matter distribution as (i) cosmological constant in the bulk and modified Chaplygin gas in the brane and (ii) positive cosmological constant in the bulk and perfect fluid (effectively a radiation equation of state at high energies) in the brane and have obtained explicit solutions in both cases.

\section{Acknowledgement:}

The authors are thankful to IUCAA, Pune, India for warm hospitality where part of the work was carried out.

\section{References:}

[1] E. R. Harison, Rev. Mod. Phys. 39862 (1967).

[2] G. F. R. Ellis and R. Maartens, Class. Quantum Grav. 21223 (2004).

[3] G. F. R. Ellis, J. Murugan and C. G. Tsagas, Class. Quantum Grav. 21233 (2004).

[4] S. Mukherjee, B. C. Paul, S. D. Maharaj and A. Beesham, gr-qc/0505103.

[5] S. Mukherjee, B. C. Paul, N. K. Dadhich, S. D. Maharaj and A. Beesham, Class. Quantum Grav. 23 6927 (2006).

[6] S. de Campo, R. Herrera and P. Labraña, JCAP 0711030 (2007).

[7] A. Banerjee, T. Bandyopadhyay and S. Chakraborty, Gravitation and Cosmology 13290 (2007); Gen. Rel. Grav. 401603 (2008).

[8] U. Debnath, Class. Quantum Grav. 25205019 (2008).

[9] L. Randall and R. Sundrum, Phys. Rev. Lett. 833770 (1999).

[10] R. Maartens, Living Rev. Rel. 71 (2004); L. Randall and R. Sundrum, Phys. Rev. Lett. 834690 (1999).

[11] B. Chang, H. Liu, L. Xu and C. Zhang, Chin. Phys. Lett. 242153 (2007). (astro-ph/0704.3768).

[12] J. -g. Hao and X. -z. Li, Phys. Rev. D 68043510 (2003); Phys. Rev. D 68083514 (2003); S. Nojiri and S. D. Odintsov, Phys. Lett. B 5711 (2003); B. Gumjudpai, T. Naskar, M. Sami and S. Tsujikawa, JCAP 0506 007 (2005). 